\title{
Effects of miRNA-455 on cardiac hypertrophy induced by pressure overload
}

\author{
CHUNTAO WU ${ }^{1}$, SHIMIN DONG ${ }^{1}$ and YONGJUN LI ${ }^{2}$ \\ ${ }^{1}$ Intensive Care Unit, The Third Hospital of Hebei Medical University; \\ ${ }^{2}$ Department of Cardiology, The Second Hospital of Hebei Medical University, Shijiazhuang, Hebei, P.R. China
}

Received November 12, 2014; Accepted February 11, 2015

DOI: $10.3892 /$ ijmm.2015.2105

\begin{abstract}
RNAs (miRNAs or miRs) are essential in cardiac hypertrophy and in the development of heart failure. In the present study, we aimed to determine whether the restoration of miRNA-455 (miR-455) gene expression in vivo aggravates hypertrophy, but protects against adverse cardiac remodeling induced by pressure overload. Cardiac hypertrophy was induced by left ventricular pressure overload in male mice subjected to transverse aortic constriction (TAC). The mice were randomly selected to receive a tail vein injection of either miR-455 or green fluorescent protein per animal at 1,8,15 and 21 days following surgery. Cardiac hypertrophy, function and remodeling were evaluated by echocardiography, catheterization, histological analysis and the examination of the expression of specific genes and cardiac apoptosis. TAC ( 2 weeks following surgery) resulted in significant cardiac hypertrophy, which was significantly aggravated by treatment with miR-455. However, miR-455 replacement therapy markedly reduced myocardial fibrosis and inhibited apoptosis, suggesting that this therapy can prevent maladaptive ventricular remodeling. miR-455 was also identified and validated to target calreticulin, a protein that is critical for cardiac development. The restoration of miR-455 gene expression may thus be a potential therapeutic strategy to reverse pressure-induced cardiac hypertrophy and prevent maladaptive cardiac remodeling through the regulation of miR-455 at different time points following hypertrophy.
\end{abstract}

\section{Introduction}

Cardiac hypertrophy is a pathological characteristic common to numerous forms of heart disease, such as hypertension, ischemic myocardial injury, diabetic cardiomyopathy, valvular dysfunction and aortic stenosis (1). Persistent hypertrophy can ultimately lead to ventricular dilatation, arrhythmia, fibrotic

Correspondence to: Dr Yongjun Li, Department of Cardiology, The Second Hospital of Hebei Medical University, 215 Hepingxi Road, Xinhua, Shijiazhuang, Hebei 050000, P.R. China E-mail: twbeer126@163.com; lyjbs2009@yeah.net

Key words: microRNA, hypertrophy/remodeling, left ventricular hypertrophy, left ventricular remodeling, calreticulin disease, heart failure and even sudden death $(2,3)$. Cardiac hypertrophy is a major risk factor in the development of heart failure, and its therapeutic reversal is associated with decreased mortality $(4,5)$. Previous studies have indicated that microRNAs (miRNAs or miRs) are essential in a number of biological processes, including differentiation, apoptosis, proliferation and development $(6,7)$. The dysregulation of miRNAs has been linked to several human diseases (7), including cardiovascular disease $(8,9)$. miRNAs are a class of highly conserved, small non-coding RNAs (approximately 23 nucleotides in length) that regulate gene expression at the post-transcriptional level (10). miRNAs inhibit gene expression by forming partial duplexes with the 3 '-untranslated region (3'-UTR) of mRNAs $(11,12)$. miRNAs function by either inhibiting mRNA translation or promoting mRNA degradation (13). Each miRNA can have numerous mRNA targets (14). In addition, a single mRNA can be targeted by different miRNAs, thereby increasing the complexity of gene regulation by miRNAs. miRNAs have been found in various organisms and are regarded as powerful regulators of gene expression and cellular phenotype. Moreover, their roles in cardiovascular biology and diseases have been an area of intense investigation. Previous studies have identified the expression patterns of miRNAs associated with cardiovascular diseases. For example, miRNA-21, miRNA-23a, miRNA-24, miRNA-133, miRNA-208/miRNA-195 and miRNA-199 have been shown to be involved in cardiac hypertrophy (15-17), miRNA-1 in arrhythmia (18), miRNA-29 and miRNA-21 in cardiac fibrosis $(19,20)$, miRNA-210 and miRNA-494 in ischemic heart disease (21) and miRNA-129 in heart failure (22). However, the association between miRNA-455 (miR-455) and cardiac hypertrophy remains unclear. In this study, using a target prediction algorithm (23), we identified calreticulin (Calr) as the putative target gene of miR-455. The mRNA sequence of Calr was predicted to contain a conserved 'seed' sequence complementary to miR-455 in the 3'-UTR (Fig. 1). Calr is closely associated with myocardial hypertrophy (24). Thus, miR-455 may be important in myocardial hypertrophy. In the present study, we established a mouse model of hypertrophy by transverse aortic constriction (TAC) in order to investigate the effects of the aberrant expression of miR-455 on cardiac hypertrophy induced by pressure overload and to elucidate the potential cellular and molecular mechanisms of action of this miRNA. 


\section{Materials and methods}

Animal models. At 10 weeks after birth, 18 Kunming male mice were provided by the Experimental Animal Center of Hebei Province, China. All experiments were carried out in compliance with the Guide for the Care and Use of Laboratory Animals (National Research Council, 1996) and were reviewed and approved by the Ethics Committee for the Use of Experimental Animals at Hebei Medical University, Shijiazhuang, China. The mice (weighing 16-18 g) were anesthetized and then subjected to either TAC or sham operation. Briefly, the transverse aorta of the anesthetized mice was constricted using a 7-0 nylon suture and was ligated using a blunted 27-gauge needle, which was later removed. The mice were examined at 2 and 4 weeks following surgery.

Ad-13 containing miR-455 precursor. The precursor of the miRNA, mmu-miR-455 (miRBase accession no. MI0004679) was produced by Invitrogen (Shanghai, China). The green fluorescent protein (GFP)-expressing vector (Invitrogen) was used as a control.

Viral delivery protocol. The mice were randomly selected to receive a tail vein injection of either miR-455 $\left(5.0 \times 10^{9} \mathrm{ifu} / \mathrm{ml}\right.$, $\mathrm{n}=12)$ or GFP $\left(1.0 \times 10^{9} \mathrm{ifu} / \mathrm{ml}, \mathrm{n}=24\right)$ at $0.1 \mathrm{ml}$ (viral genomes) per animal at $1,8,15$ and 21 days following surgery. Echocardiographic measurements were taken at baseline and at 2 and 4 weeks after TAC. Invasive hemodynamic measurements were also obtained at 2 and 4 weeks after TAC, and the the animals were then sacrificed by cervical dislocation.

Echocardiography and hemodynamic measurements. Transthoracic echocardiography was performed using a $30 \mathrm{MHz}$ high-frequency scan head (VisualSonics Vevo770; VisualSonics Inc., Toronto, ON, Canada). All measurements were averaged for 5 consecutive cardiac cycles. Aortic blood pressure (BP), left ventricular end-systolic pressure (LVESP) and left ventricular end-diastolic pressure (LVEDP) were measured. Briefly, a micromanometer catheter (Millar 1.4F, SPR-835; Millar Instruments, Inc., Houston, TX, USA) was inserted through the right common carotid artery into the aorta and carefully introduced into the left ventricle (LV). The transducer was connected to a Power Laboratory system (ADInstruments, Castle Hill, Australia) and BP, LVESP and LVEDP were recorded.

Morphological and histological analyses. The mice were sacrificed by cervical dislocation, and their hearts were excised at 2 and 4 weeks after TAC. For global morphometry, the hearts were perfused with phosphate-buffered saline followed by $4 \%$ paraformaldehyde. For histological analysis, the heart tissues were fixed in $10 \%$ formalin, embedded in paraffin or frozen in liquid nitrogen, sectioned at $4 \mathrm{~mm}$ thickness, and then stained with hematoxylin and eosin and Masson's trichrome (Senbeijia Co., Ltd., Jiangsu, China). The heart tissue morphological characteristics and the differences between the 3 experimental groups (sham-operated group, the group subjected to TAC and injected with GFP and the group subjected to TAC and injected with miR-455) were observed under a microscope (Nikon, Tokyo, Japan). The extent of fibrosis was evaluated by measuring the Masson's
miR-455 : 5' UAUGUGCCUUUgGacuacaucG 3'

\section{Calr : 3'GgaAuCCCGACCUGACUCCGg 5'}

Figure 1. Bioinformatics analysis identified Calr as a putative target gene of miR-455. Calr, calreticulin.

trichrome-stained area in the entire left ventricular wall. All measurements were acquired using an automated image analysis system (Motic6.0; Motic, Xiamen, China).

In situ detection of myocardial apoptosis. The apoptotic cells were fixed and permeabilized. Subsequently, the cells were incubated with $50 \mu$ l terminal deoxynucleotidyl transferase-mediated dUTP nick-end labeling (TUNEL) reaction mixture (In Situ Cell Death Detection kit, POD; Roche, Shanghai, China) and kept for $60 \mathrm{~min}$ in a wet box. After washing, the label incorporated at the damaged sites of the DNA was marked by an anti-fluorescein antibody conjugated with the reporter enzyme, peroxidase. After washing to remove the unbound enzyme conjugate, the POD retained in the immune complex was visualized by a substrate reaction. The TUNEL-positive cells were imaged under a microscope at a magnification of $\mathrm{x} 400$ (Nikon) and 3 horizons were randomly selected in each slice. The TUNEL-positive cells were counted using a digital medical image analysis system (Motic6.0; Motic). The results were expressed as the number of TUNEL-positive cells $/ 10^{2}$ cardiomyocytes.

Reverse transcription-quantitative polymerase chain reaction (RT-qPCR) of mature miRNAs. Total RNA was extracted from the LV tissues of the mice using TRIzol reagent (Invitrogen) and reverse transcribed into complementary DNA (cDNA) using EasyScript First-Strand cDNA Synthesis SuperMix (TransGen, Beijing, China) following the manufacturer's instructions. Mature miR-455 expression was quantified by RT-qPCR using the All-in-One miRNA qRT-PCR Detection system following the manufacturer's instructions (GeneCopoeia, Guangdong, China). The primers used for PCR were as follows: miR-455 forward, ATGTGCCTTTGGACTACATCGAA; and U6 forward, TCGTGAAGCGTTCCATATTTTTAA; consensus primer sequence, TTACTACGTCATGACTAGTAA. The program was initially run for $10 \mathrm{~min}$ at $95^{\circ} \mathrm{C}$, followed by 40 cycles of $10 \mathrm{sec}$ at $95^{\circ} \mathrm{C}, 27 \mathrm{sec}$ at $60^{\circ} \mathrm{C}$ and $27 \mathrm{sec}$ at $72^{\circ} \mathrm{C}$. Gene expression levels were normalized to the U6 rRNA endogenous control and fold changes were calculated using the $\Delta \Delta \mathrm{Ct}$ method.

For qPCR, the transcribed complementary DNA was then subjected to qPCR analysis using a Bio-Rad IQ5 multicolor detection system (Bio-Rad, Hercules, CA, USA). A comparative cycle threshold method was used to determine the relative quantification of RNA expression. All PCR reactions were performed at least in triplicate. Atrial natriuretic factor (Anf), skeletal muscle alpha-actin (Acta1), $\beta$-myosin heavy chain (Myh7), transforming growth factor $\beta-1(\operatorname{Tgf} \beta 1)$, connective tissue growth factor (Ctgf), Calr, glucose-regulated protein 78 (GRP78) and $\beta$-actin were amplified using their specific primers (Table I). The program was initially run for $30 \mathrm{sec}$ at $95^{\circ} \mathrm{C}$, followed by 40 cycles of $5 \mathrm{sec}$ at $95^{\circ} \mathrm{C}, 20 \mathrm{sec}$ at $60^{\circ} \mathrm{C}$ and $20 \mathrm{sec}$ at $72^{\circ} \mathrm{C}$. 


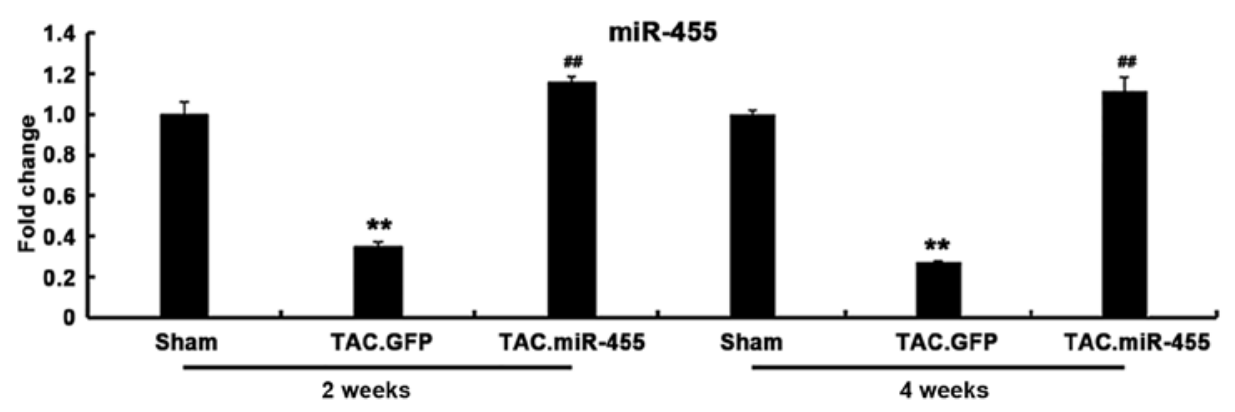

Figure 2. In vivo restoration of mature miR-455 expression. The in vivo restoration of mature miR-455 expression evaluated as a fold change at 2 and 4 weeks after gene transfer was assessed by RT-qPCR. miR-455 expression levels were normalized to those of U6 rRNA. Values are expressed as the means \pm SE. Significance of differences: ${ }^{* *} \mathrm{P}<0.01$ vs. sham-operated group (sham); ${ }^{\# /} \mathrm{P}<0.01$ vs. TAC.GFP $(\mathrm{TAC}+\mathrm{GFP})$. TAC, transverse aortic constriction; GFP, green fluorescent protein.

Table I. Primers for quantitative polymerase chain reaction.

\begin{tabular}{ll}
\hline Gene names & \multicolumn{1}{c}{ Primer sequences } \\
\hline Anf & F: GCTCCTTCTCCATCACCCTG \\
& R: ACCGGCATCTTCTCCTCCA \\
Acta1 & F: TGAGCGTGGCTATTCCTTCG \\
& R: CCGCAGACTCCATACCGATAA \\
Myh7 & F: GCCAACACCAACCTGTCCAAGTTC \\
& R: TTCAAAGGCTCCAGGTCTCAGGGC \\
Tgf $\beta 1$ & F: CCCGAGTGTGGAAGATGAGAA \\
& R: AACCTGAAAGCAGCCCTTCTG \\
Ctgf & F: AATCGCCAAGCCTGTCAAGT \\
& R: CCCAGGACAGTTGTAATGGCA \\
Calr & F: TCTGTCCCTCCCTTTCTCCA \\
& R: AGCTGTGCTAGAACTGGCTGC \\
GRP78 & F: TCTGGTTGGCGGATCTACTC \\
& R: TCTTTTGCAGGGGTCGTTC \\
& F: AGCGTGGCTACAGCTTCACC \\
& R: CCGCTCGTTGCCAATAGTG
\end{tabular}

Anf, atrial natriuretic factor; Acta1, skeletal muscle $\alpha$-actin; Myh7, $\beta$-myosin heavy chain; Tgf $\beta 1$, transforming growth factor $\beta 1$; Ctgf, connective tissue growth factor; Calr, calreticulin; GRP78, glucoseregulated protein 78 ; F, forward; $R$, reverse.

Western blot analysis. Total protein was obtained from the ventricular myocardial tissues using tissue homogenates, centrifugation and heat denaturation. The protein lysates were electrophoresed and separated by $6-12 \%$ sodium dodecyl sulfate-polyacrylamide gel electrophoresis (SDS-PAGE) and transferred onto PVDF membranes (Millipore Immobion-P; BioSharp, Anhui, China). The membranes were blocked with $5 \%$ skim milk at room temperature for $1 \mathrm{~h}$, and then incubated overnight at $4^{\circ} \mathrm{C}$ with primary antibodies, including rabbit antiBcl-2 (BA0412; 1:500), rabbit anti-Bax (BA0315-2; 1:500), rabbit anti-Calr (BM1798; 1:500), rabbit anti-GRP78 (BA2042; 1:500) (all from Boster Biotechnology, Inc., Wuhan, China), and rabbit anti-glyceraldehyde 3-phosphate dehydrogenase (GAPDH) (AP0063; 1:5,000; BioWorld, Inc., Jiangsu, China). The membranes were then incubated with IRDye800-conjugated secondary antibodies (1:20,000; Rockland, Inc., Gilbertsville, PE, USA) at room temperature for $1 \mathrm{~h}$. The Odyssey double color infrared laser imaging system (LI-COR; Lincoln, NE, USA) was used to detect the antigen-antibody complexes in a western blotting detection system (Bio-Rad). The results were expressed as density values normalized to GAPDH.

Statistical analysis. Data are expressed as the means \pm standard error of the mean. The underlying assumption of normal distribution was investigated by performing a Kolmogorov-Smirnoff normality test and a normal probability plot test. Statistical significance between 2 groups was examined by a t-test for normal distribution and by one-way ANOVA for multi-group comparisons. When the ANOVA results were significant, the differences among individual groups were determined using the Bonferroni post hoc test. A value of $\mathrm{P}<0.05$ was considered to indicate a statistically significant difference.

\section{Results}

miR-455 gene expression in vivo. The gene expression of miR-455 was significantly decreased in the GFP-treated hearts, but was significantly increased in the miR-455-treated hearts after TAC. The expression of the mature miR-455 in the hypertrophied hearts induced by pressure overload was restored by Ad-mediated miR-455 gene transfer in vivo (Fig. 2).

miR-455 aggravates cardiac hypertrophy in mice 2 weeks after TAC. The effects of miR-455 on cardiac hypertrophy at 2 weeks after TAC were investigated. At this time point, all the experimental mice survived, and some were subjected to hemodynamic and echocardiographic examinations. BP and LVESP were similarly elevated, whereas LVEDP was not altered in all the mice examined. TAC induced significant cardiac hypertrophy, as characterized by an increased left ventricular wall thickness [left ventricular anterior wall thickness at end diastole (LVAWd)], decreased left ventricular cavity dimension [left ventricular internal dimension at end diastole (LVIDd)], increased heart-to-body weight ratio and an expanded crosssectional area of cardiomyocytes in the GFP-treated mice. All these characteristics were significantly aggravated by treatment with miR-455 2 weeks after TAC (Fig. 3).

miR-455 gene transfer preserves cardiac adaptation and function at 4 weeks after TAC. In the GFP-treated mice, BP, LVESP, the heart-to-body weight ratio and the cardiomyocyte cross-sectional area were not altered at 4 weeks after TAC 

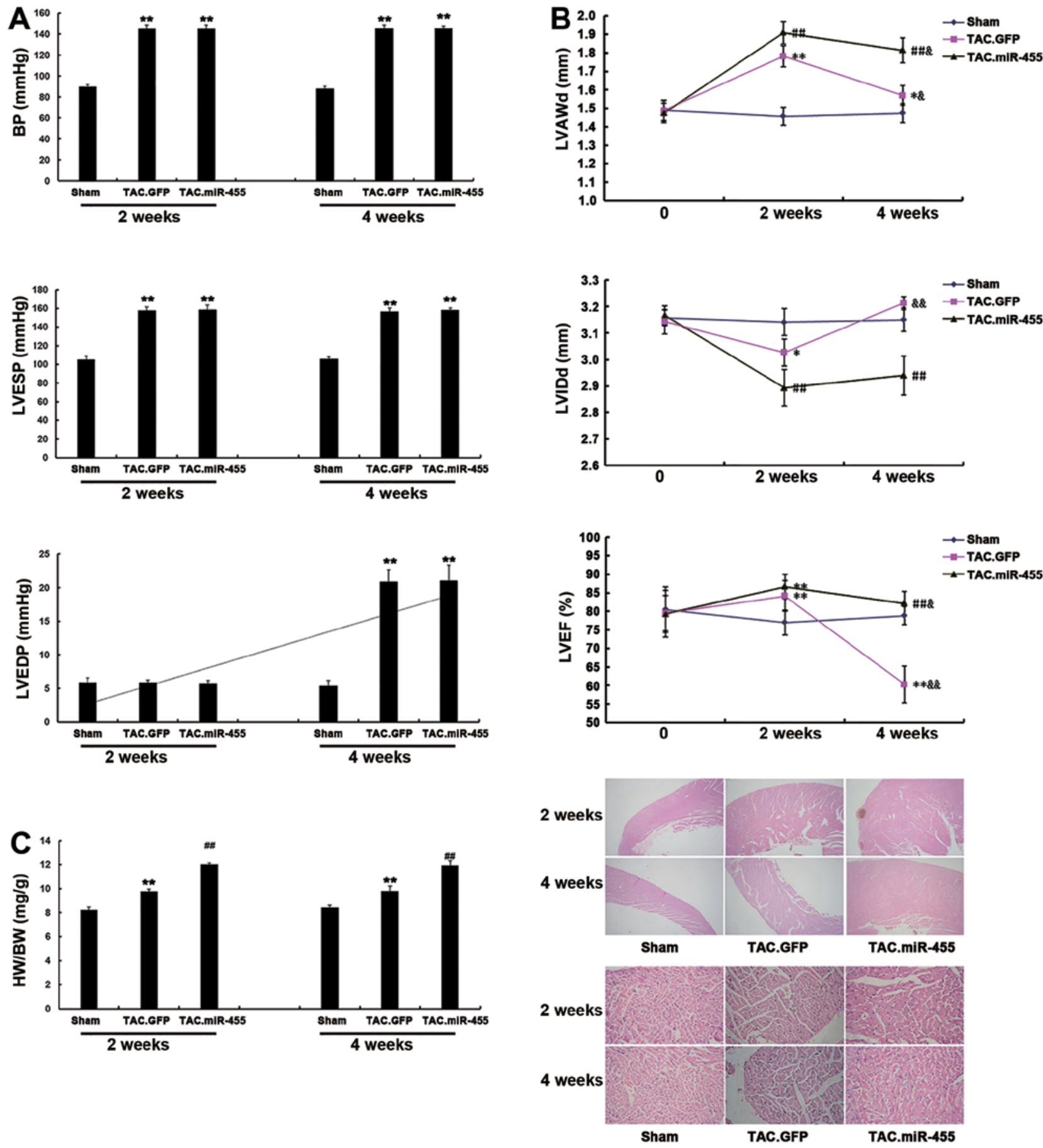

Figure 3. Effects of miR-455 on hemodynamic, echocardiographic and pathological parameters at 2 weeks and 4 weeks after transverse aortic constriction (TAC). Mice were administered with miR-455 or GFP and subjected to sham operation or TAC. (A) Representative quantitative analyses of blood pressure (BP) and left ventricular pressure (LVP) are shown. (B) Representative quantitative analyses of left ventricular anterior wall thickness at end diastole (LVAWd), left ventricular internal dimension at end diastole (LVIDd) and left ventricular ejection fraction (LVEF) are shown. (C) Representative cardiomyocyte images (hematoxylin and eosin-stained) of left ventricular wall (magnification, x100) and cross-sectional area (magnification, x400) and the ratio of heart weight to body weight $(\mathrm{HW} / \mathrm{BW})$ are shown. Values are expressed as the means $\pm \mathrm{SE}$. Significance of differences: ${ }^{*} \mathrm{P}<0.05$ and ${ }^{* *} \mathrm{P}<0.01$ vs. sham-operated group (sham); ${ }^{\# \#} \mathrm{P}<0.01$ vs. TAC.GFP $(\mathrm{TAC}+\mathrm{GFP}) ;{ }^{\&} \mathrm{P}<0.05$ and ${ }^{\& \&} \mathrm{P}<0.01$ vs. respective mice at 2 weeks after TAC. GFP, green fluorescent protein.

as compared with the levels observed at 2 weeks after TAC. However, at 4 weeks after TAC, GFP treatment elevated LVEDP, reduced left ventricular wall thickness (LVAWd), enlarged left ventricular cavity dimension (LVIDd) and lowered left ventricular contractility (ejection fraction) (Fig. 3). These results indicate significant cardiac remodeling with impaired cardiac function in the mice at 4 weeks after TAC. Treatment with miR-455 reduced LVAWd, but did not enlarge left ventricular cavity dimension (LVIDd) and did not lower LV contractility. Thus, the miR-455-treated hearts maintained a state of myocardial hypertrophy. These results suggest that miR-455 gene transfer is effective in the prevention of cardiac remodeling and dysfunction during a 4-week period of pressure overload.

miR-455 modulates the expression of molecular markers of cardiac hypertrophy. The effects of miR-455 treatment on 

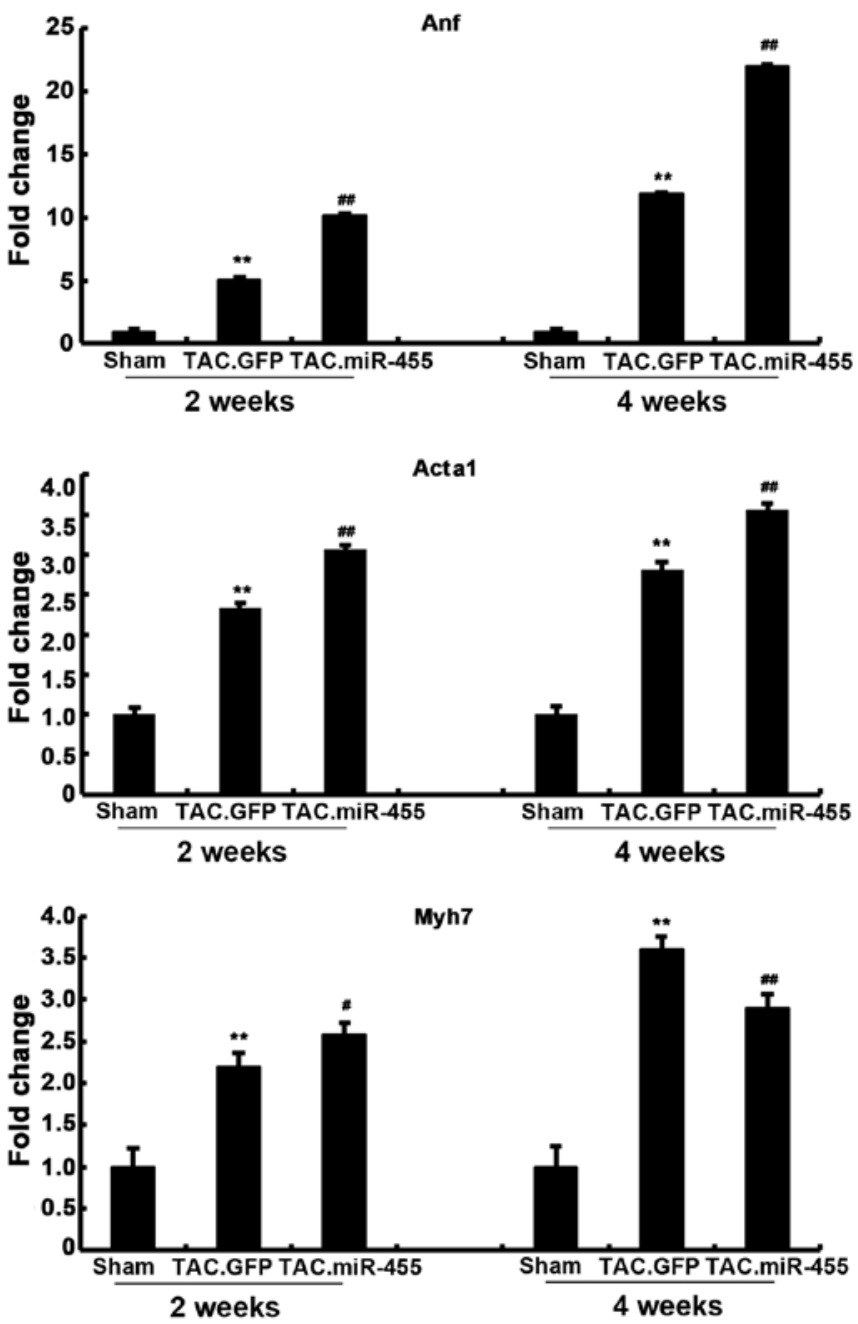

Figure 4. Expression levels of myocardial hypertrophy genes. Relative mRNA expression levels of Anf, Actal and Myh7 were evaluated as the fold change at 2 and 4 weeks after gene transfer. Values are expressed as the means $\pm \mathrm{SE}$. Significance of differences: ${ }^{* *} \mathrm{P}<0.01$ vs. sham-operated group (sham); ${ }^{*} \mathrm{P}<0.05$ and ${ }^{\# \#} \mathrm{P}<0.01$ vs. TAC.GFP (TAC + GFP); Anf, atrial natriuretic factor; Acta1, skeletal muscle alpha-actin; Myh7, $\beta$-myosin heavy chain; TAC, transverse aortic constriction; GFP, green fluorescent protein.

molecular abnormalities associated with pathological hypertrophy were investigated. We assessed the expression of the hypertrophic fetal genes, Anf, Actal and Myh7 at 2 and 4 weeks after gene transfer (Fig. 4). The pressure overload-induced hypertrophy in the GFP-treated group was associated with the re-induction of the 'fetal gene program', which was characterized by a significant increase in the mRNA expression levels of Anf, Myh7 and Acta1, as compared with those in the sham-operated group. Furthermore, the expression levels of these 3 genes significantly increased in the miR-455-treated mouse hearts compared with the GFP-treated mouse hearts. That is, the expression levels of Anf, Myh7 and Actal were significantly upregulated by the pressure overload in the GFP-treated hearts, and these hypertrophic responses were significantly aggravated after gene transfer. However, the expression of Myh7 was higher in the GFP-treated mice than in the miR-455-treated mice at 4 weeks. sion of molecular markers of cardiac fibrosis at 4 weeks after
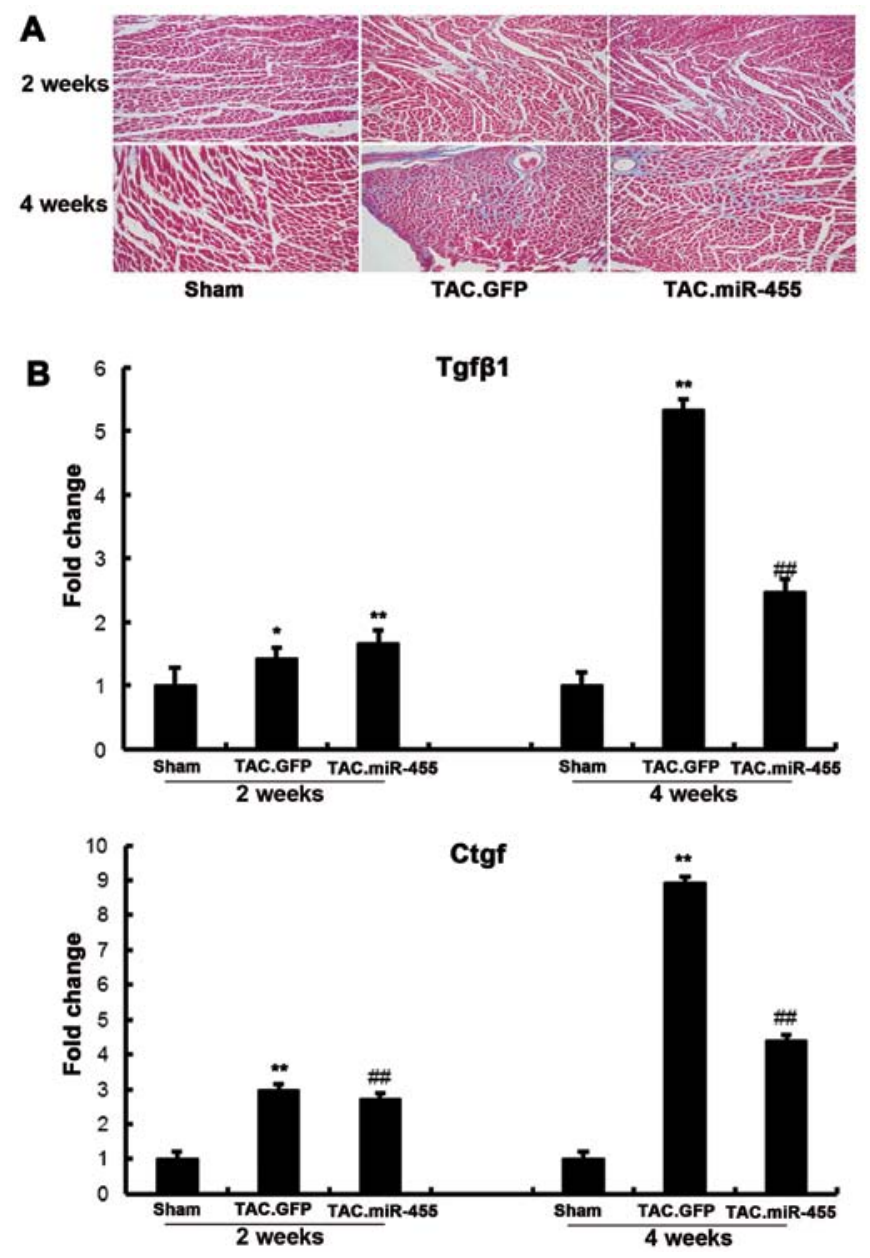

Figure 5. Effects of miR-455 on myocardial fibrosis in mice after TAC. Mice were treated with GFP or miR-455 and subjected to sham operation or TAC. (A) Images of myocardial fibrosis (Masson-stained, magnification, x200) are shown. Blue areas indicate fibrotic staining. (B) Relative mRNA expression levels of Tgf $\beta 1$ and $\mathrm{Ctgf}$ genes were evaluated as the fold change at 2 and 4 weeks after gene transfer. Values are expressed as the means \pm SE. Significance of differences: ${ }^{*} \mathrm{P}<0.05$ and ${ }^{* *} \mathrm{P}<0.01$, vs. sham-operated group (sham); ${ }^{\# \#} \mathrm{P}<0.01$ vs. TAC.GFP (TAC + GFP). Tgf $\beta 1$, transforming growth factor $\beta-1$; Ctgf, connective tissue growth factor; TAC, transverse aortic constriction; GFP, green fluorescent protein.

TAC. Considering that cardiac fibrosis and apoptosis are prominent features in the transition from compensatory hypertrophy to heart failure, we investigated the potential involvement of the restoration of miR-455 in the regulation of cardiac ECM remodeling and apoptosis. Fibrosis is a pathological characteristic of cardiac adaptation to stress, where the proliferation of fibroblasts and the increased deposition of extracellular matrix (ECM) components result in myocardial stiffness and diastolic dysfunction (24). Previous studies have demonstrated that miRNAs play central roles in controlling cardiac fibrosis and pathological LV remodeling $(20,25,26)$. Histological examination of the left ventricular sections by Masson's trichrome staining and the subsequent quantification of the fibrotic area revealed that TAC significantly increased interstitial fibrosis in the GFP-treated hearts as compared with the sham-operated hearts. By contrast, treatment with miR-455 significantly decreased fibrosis (Fig. 5).

The mRNA expression levels of $\operatorname{Tgf} \beta 1$ and Ctgf significantly increased in the GFP-treated hearts as compared with the sham-operated hearts (Fig. 6). However, these increased 

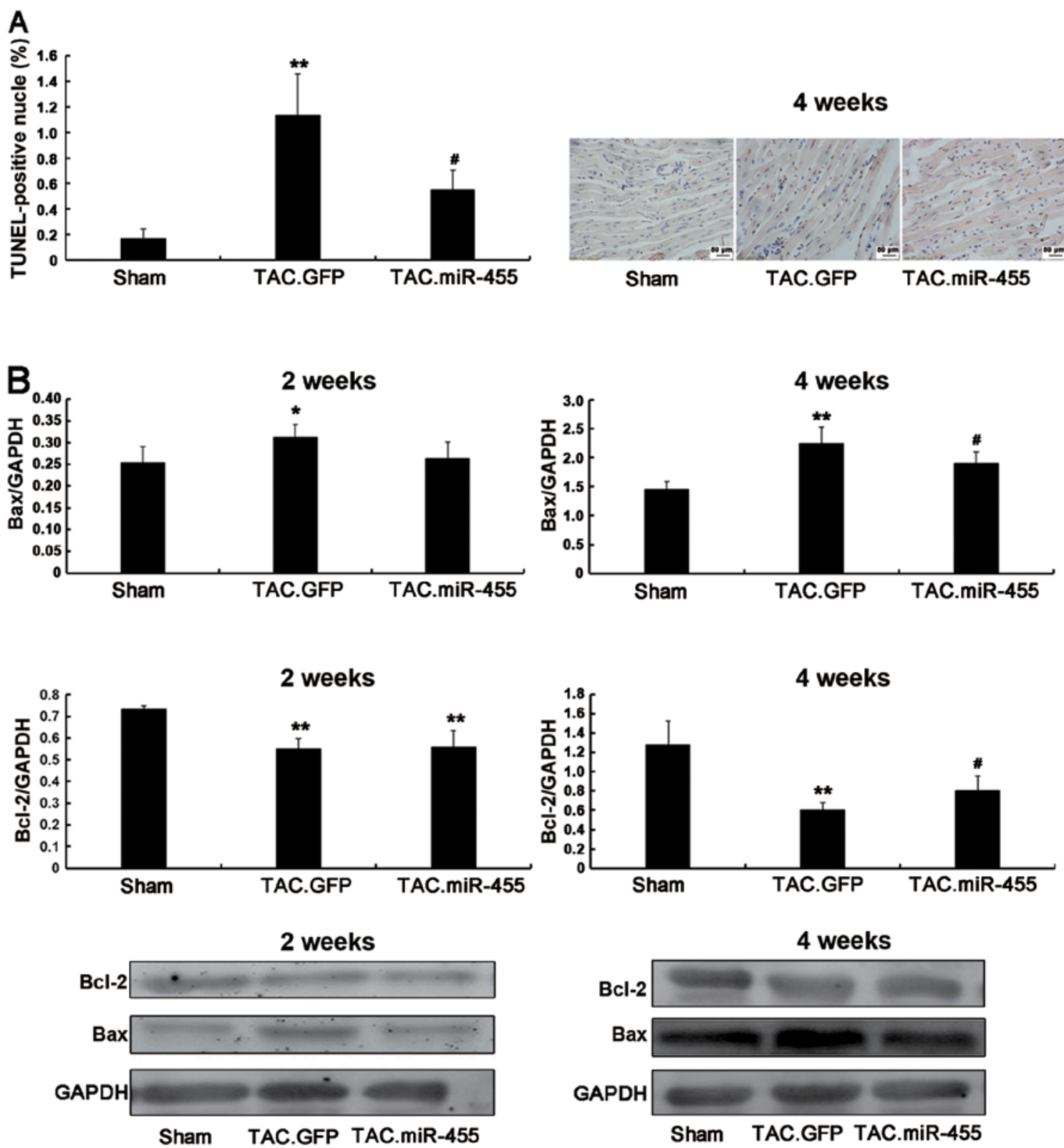

Figure 6. Effects of miR-455 on myocardial apoptosis in mice after TAC. Mice were treated with GFP or miR-455 and subjected to sham operation or TAC (A) Apoptosis. Representative images of staining with TUNEL in left ventricular tissues are shown. Purple indicates TUNEL-positive nuclei. Quantification of apoptosis was performed in 20 fields randomly selected from the LV wall for each section. Five sections from each heart were measured. Values were expressed as the number of TUNEL-positive cells $/ 10^{2}$ cardiomyocytes. (B) Protein expression levels of Bax and Bcl-2 normalized against GAPDH levels in the myocardium. Values are expressed as the means \pm SE. Significance of differences: ${ }^{*} \mathrm{P}<0.05$ and ${ }^{* *} \mathrm{P}<0.01$ vs. sham-operated group (sham); ${ }^{\#} \mathrm{P}<0.05$ and ${ }^{\# \#} \mathrm{P}<0.01$ vs. TAC.GFP $(\mathrm{TAC}+\mathrm{GFP})$. TAC, transverse aortic constriction; GFP, green fluorescent protein

levels of myocardial fibrosis-related genes following pressure overload were significantly decreased in the mice treated with miR-455.

miR-455 inhibits heart myocardial apoptosis. In response to pressure overload, cardiomyocyte apoptosis may further contribute to the transition from left ventricular hypertrophy to heart failure (27). Using western blot analysis, we quantified the protein expression of the anti-apoptotic gene, Bcl-2, and the pro-apoptotic gene, Bax (Fig. 6). Compared to treatment with GFP, treatment with miR-455 significantly increased $\mathrm{Bcl}-2$ expression and decreased Bax expression. Consequently, the Bcl-2/Bax ratio, an important marker of myocardial cell survival probability (27), was significantly increased in the
miR-455-treated hearts as compared with the GFP-treated hearts (Fig. 6).

Calr is a direct target of miR-455 that is involved in miR-455-mediated effects in mouse hearts after TAC. Endoplasmic reticulum (ER) stress occurs during myocardial hypertrophy $(28,29)$. The sarcoplasmic reticulum is a principal subcellular organelle that regulates the calcium homeostasis, protein synthesis and the apoptosis of cardiomyocytes. ER stress triggers calcium homeostasis imbalance and abnormal functional protein formation; these phenomena are accompanied by upregulated ER chaperones, such as Calr and GRP78 (30-32). However, the association between miRNA-455 and Calr during cardiac hypertrophy remains unclear. Therefore, we evaluated 
A 2 weeks
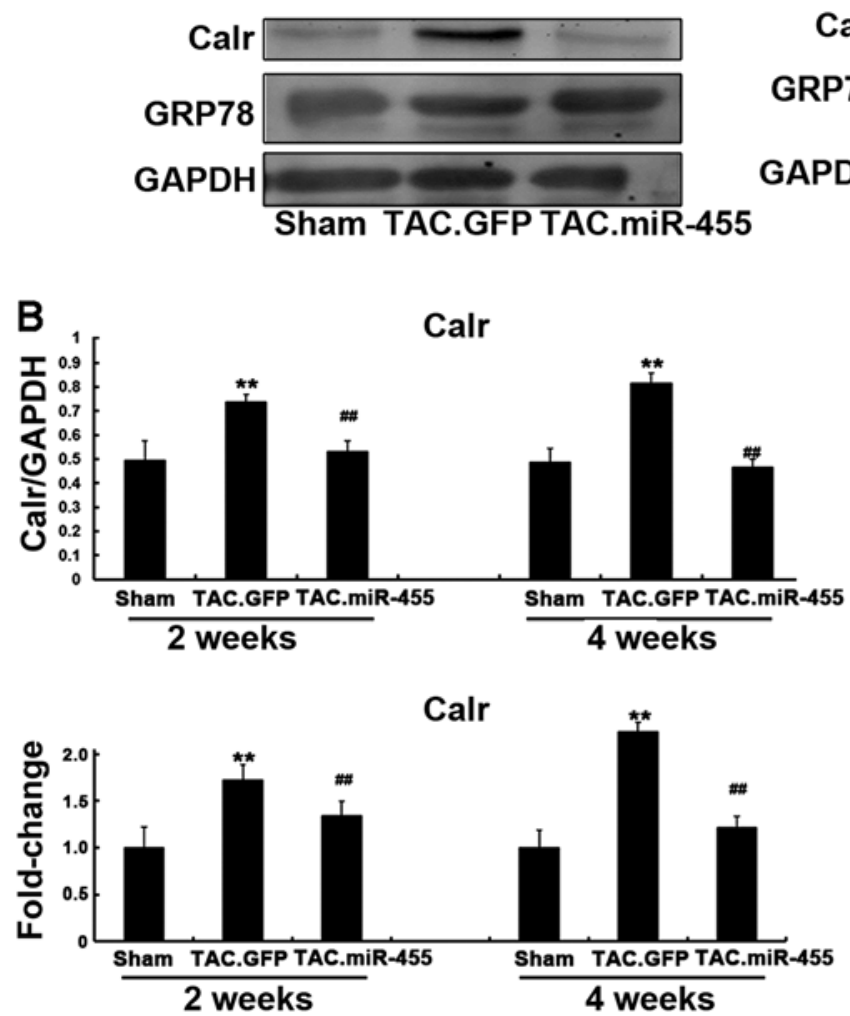

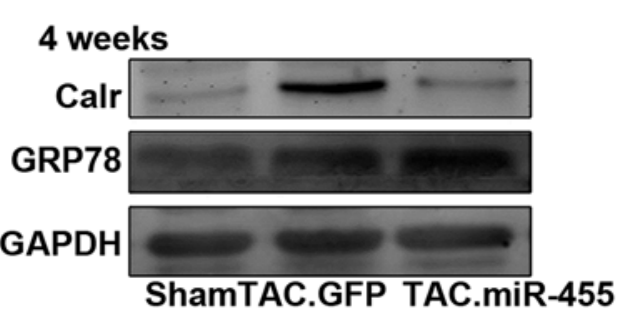

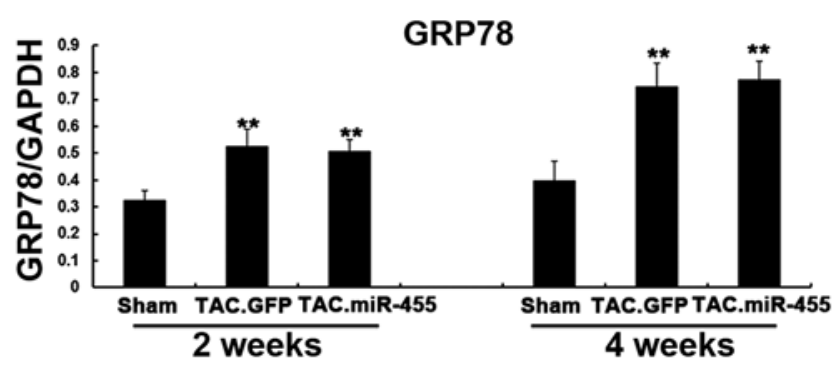

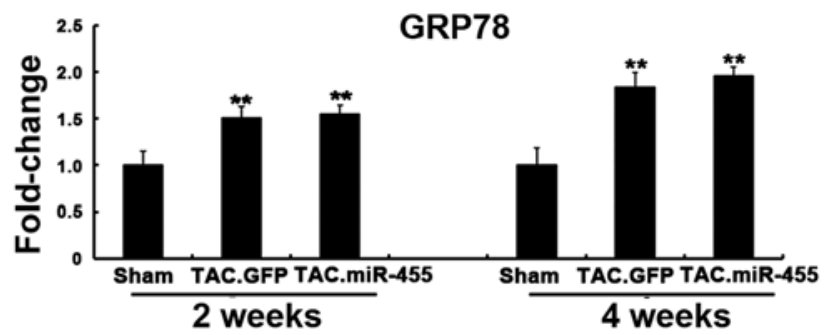

Figure 7. Identification and validation of miR-455 direct target gene. (A) Representative western blots of Calr and GRP78 protein expression. (B) Relative mRNA gene expression levels of Calr and GRP78 evaluated as the fold change at 2 and 4 weeks after gene transfer. Values are expressed as the means \pm SE. Significance of differences: ${ }^{* *} \mathrm{P}<0.01$ vs. sham-operated group (sham); ${ }^{\# \#} \mathrm{P}<0.01$ vs. TAC.GFP (TAC + GFP). TAC, transverse aortic constriction; GFP, green fluorescent protein; Calr, calreticulin.

whether the expression levels of Calr and GRP78 are regulated by miR-455 in vivo in hypertrophy induced by pressure overload. Western blot analysis revealed that the protein expression levels of both Calr and GRP78 significantly increased in the left ventricular tissue of the GFP-treated mice as compared with the sham-operated animals. However, Calr was downregulated and GRP78 was upregulated with the upregulation of miR-455 in the left ventricular tissue of the miR455-treated mice (Fig. 7). These results indicate that Calr and not GRP78 is the target of miR-455. miRNAs can also degrade the mRNA of their targets $(33,34)$. The results of RT-qPCR revealed that miR-455 significantly decreased the mRNA expression level of Calr, but did not alter the mRNA expression level of GRP78 as compared with GFP (Fig. 7).

\section{Discussion}

To the best of our knowledge, this study is the first to demonstrate that miR-455 is downregulated in pressure overload-induced cardiac hypertrophy in vivo and that this downregulation increases the mRNA and protein levels of Calr, the predicted target of miR-455. This study assessed the short-term and long-term effects of miR-455 gene transfer in pressure overload-induced cardiac hypertrophy in vivo using a cardiotropic Ad-13 vector that efficiently transduced cardiac tissues. This study is also the first to reveal the short-term and long-term effects of miR-455 on pressure overload-induced cardiac hypertrophy. A number of fetal genes, such as Anf,
Acta1 and Myh7, are re-expressed during the cardiac hypertrophic response (35). These 3 genes were upregulated in the mice following aortic coarctation, particularly in the miR455-treated mice. A transition occurred from left ventricular hypertrophy to heart failure in response to long-term pressure overload. Cardiac fibrosis and apoptosis are prominent characteristics in the transition from compensatory hypertrophy to heart failure. Cardiac fibrosis and apoptosis were alleviated in the miR-455-treated mice. The normalization of miR-455 gene expression levels, which were downregulated during hypertrophy, aggravated cardiac hypertrophy in the short term, but attenuated pathological remodeling in the long term.

The different effects of miR-455 on myocardial hypertrophy in the short and long term may be related to its target gene, Calr. The conditions in the ER must be optimal to facilitate the efficient synthesis and folding of most secreted membrane proteins; suboptimal conditions lead to improper protein folding and eventual ER stress (36). Initially, ER stress triggers protective aspects of the conserved signaling program known as the unfolded protein response (UPR), and these aspects are oriented toward restoring the ER environment (37-39). However, apoptotic aspects of the UPR will ensue if the stress continues and ER protein folding is not restored $(40,41)$. Calr, a $\mathrm{Ca}^{2+}$-binding protein of the ER, is an important chaperone that works in conjunction with calnexin and protein disulfide isomerase. It affects intracellular $\mathrm{Ca}^{2+}$ homeostasis through its $\mathrm{Ca}^{2+}$ storage capacity and its effects on both the SERCA pumps and inositol 1,4,5-trisphosphate receptors $(42,43)$. Our 
findings on the different effects of miR-455 are in agreement with other data demonstrating the effects of Calr on cardiomyocytes $(28,30)$. Thus, the myocardial state during the application of miR-455 for the treatment of myocardial hypertrophy is highly important. This study found no evidence to prove that miR-455 directly influences the different genes. The change observed may be due to an indirect effect of miR-455 on the different genes. Furthermore, this change may differ when hypertrophy results from other causes than TAC with or without miR-455 transfection.

In conclusion, the Ad-13-mediated normalization of miR-455 expression aggravates the hypertrophic phenotype, but attenuates the progressive deterioration of left ventricular function. The restoration or downregulation of miR-455 at different time periods may lead to a pioneering therapeutic strategy to reverse cardiac hypertrophy and alleviate function deterioration.

\section{References}

1. Ho YL, Wu CC, Lin LC, et al: Assessment of the coronary artery disease and systolic dysfunction in hypertensive patients with the dobutamine-atropine stress echocardiography: effect of the left ventricular hypertrophy. Cardiology 89: 52-58, 1998.

2. Aaronson KD and Sackner-Bernstein J: Risk of death associated with nesiritide in patients with acutely decompensated heart failure. JAMA 296: 1465-1466, 2006.

3. Catalucci D, Latronico MV, Ellingsen O and Condorelli G: Physiological myocardial hypertrophy: how and why. Front Biosci 13: 312-324, 2008.

4. Koren MJ, Devereux RB, Casale PN, Savage DD and Laragh JH: Relation of left ventricular mass and geometry to morbidity and mortality in uncomplicated essential hypertension. Ann Intern Med 114: 345-352, 1991.

5. McKinsey TA and Kass DA: Small-molecule therapies for cardiac hypertrophy: moving beneath the cell surface. Nat Rev Drug Discov 6: 617-635, 2007.

6. Bartel DP and Chen CZ: Micromanagers of gene expression: the potentially widespread influence of metazoan microRNAs. Nat Rev Genet 5: 396-400, 2004.

7. Kloosterman WP and Plasterk RH: The diverse functions of microRNAs in animal development and disease. Dev Cell 11: 441-450, 2006.

8. Divakaran V and Mann DL: The emerging role of microRNAs in cardiac remodeling and heart failure. Circ Res 103: 1072-1083, 2008.

9. van Rooij E and Olson EN: MicroRNAs: powerful new regulators of heart disease and provocative therapeutic targets. J Clin Invest 117: 2369-2376, 2007.

10. Bartel DP: MicroRNAs: target recognition and regulatory functions. Cell 136: 215-233, 2009.

11. van Rooij E: The art of microRNA research. Circ Res 108: 219-234, 2011.

12. Bartel DP: MicroRNAs: genomics, biogenesis, mechanism, and function. Cell 116: 281-297, 2004.

13. van Rooij E, Marshall WS and Olson EN: Toward microRNAbased therapeutics for heart disease: the sense in antisense. Circ Res 103: 919-928, 2008

14. Small EM, Frost RJ and Olson EN: MicroRNAs add a new dimension to cardiovascular disease. Circulation 121: 1022-1032, 2010.

15. Care A, Catalucci D and Felicetti F: MicroRNA-133 controls cardiac hypertrophy. Nat Med 13: 613-618, 2007.

16. van Rooij E, Sutherland LB, Liu N, et al: A signature pattern of stress-responsive microRNAs that can evoke cardiac hypertrophy and heart failure. Proc Natl Acad Sci USA 103: 18255-18260 2006.

17. van Rooij E, Sutherland LB, Qi X, Richardson JA, Hill J and Olson EN: Control of stress-dependent cardiac growth and gene expression by a microRNA. Science 316: 575-579, 2007.

18. Yang B, Lin $\mathrm{H}$ and Xiao J: The muscle-specific microRNA miR-1 regulates cardiac arrhythmogenic potential by targeting GJA1 and KCNJ2. Nat Med 13: 486-491, 2007.
19. Thum T, Gross C and Fiedler J: MicroRNA-21 contributes to myocardial disease by stimulating MAP kinase signalling in fibroblasts. Nature 456: 980-984, 2008.

20. van Rooij E, Sutherland LB, Thatcher JE, et al: Dysregulation of microRNAs after myocardial infarction reveals a role of miR-29 in cardiac fibrosis. Proc Natl Acad Sci USA 105: 13027-13032, 2008.

21. Hu S, Huang M and Li Z: MicroRNA-210 as a novel therapy for treatment of ischemic heart disease. Circulation 122: S124-S131, 2010.

22. Thum T, Galuppo P and Wolf C: MicroRNAs in the human heart: A clue to fetal gene reprogramming in heart failure. Circulation 116: 258-267, 2007.

23. Betel D, Wilson M, Gabow A, Marks DS and Sander C: The microRNA.org resource: targets and expression. Nucleic Acids Res 36: D149-D153, 2008.

24. Papp S, Dziak E, Kabir G, Backx P, Clement S and Opas M: Evidence for calreticulin attenuation of cardiac hypertrophy induced by pressure overload and soluble agonists. Am J Pathol 176: 1113-1121, 2010.

25. Creemers EE and Pinto YM: Molecular mechanisms that control interstitial fibrosis in the pressure-overloaded heart. Cardiovasc Res 89: 265-272, 2011.

26. Duisters RF, Tijsen AJ, Schroen B, et al: miR-133 and miR-30 regulate connective tissue growth factor: implications for a role of microRNAs in myocardial matrix remodeling. Circ Res 104: 170-178, 2009.

27. Condorelli G, Morisco C, Stassi G, et al: Increased cardiomyocyte apoptosis and changes in proapoptotic and antiapoptotic genes bax and bcl-2 during left ventricular adaptations to chronic pressure overload in the rat. Circulation 99: 3071-3078, 1999.

28. Okada K, Minamino T and Tsukamoto Y: Prolonged endoplasmic reticulum stress in hypertrophic and failing heart after aortic constriction: possible contribution of endoplasmic reticulum stress to cardiac myocyte apoptosis. Circulation 110: 705-712, 2004.

29. Brostrom MA, Mourad F and Brostrom CO: Regulated expression of GR P78 during vasopressin-induced hypertrophy of heart-derived myocytes. J Cell Biochem 83: 204-217, 2001.

30. Lee KH, Lee $\mathrm{N}$, Lim S, et al: Calreticulin inhibits the MEK1,2-ERK1,2 pathway in alpha 1-adrenergic receptor/ Gh-stimulated hypertrophy of neonatal rat cardiomyocytes. J Steroid Biochem Mol Biol 84: 101-107, 2003.

31. Xu FF, Liu XH and Zhu XM: Calreticulin upregulation induced by hypoxic preconditioning relieves oxidative stress injury in rat cardiomyocytes. Sheng Li Xue Bao 60: 29-37, 2008 (In Chinese).

32. Liu XH: Endoplasmic reticulum stress and myocardial hypertrophy. Sheng Li Xue Bao 61: 9-14, 2009 (In Chinese).

33. Guo H, Ingolia NT, Weissman JS and Bartel DP: Mammalian microRNAs predominantly act to decrease target mRNA levels. Nature 466: 835-840, 2010.

34. Lim LP, Lau NC, Garrett-Engele P, et al: Microarray analysis shows that some microRNAs downregulate large numbers of target mRNAs. Nature 433: 769-773, 2005.

35. Heineke J and Molkentin JD: Regulation of cardiac hypertrophy by intracellular signalling pathways. Nat Rev Mol Cell Biol 7: 589-600, 2006

36. Glembotski CC: Endoplasmic reticulum stress in the heart. Circ Res 101: 975-984, 2007.

37. Kaufman RJ: Stress signaling from the lumen of the endoplasmic reticulum: coordination of gene transcriptional and translational controls. Genes Dev 13: 1211-1233, 1999.

38. Austin RC: The unfolded protein response in health and disease. Antioxid Redox Signal 11: 2279-2287, 2009.

39. Glembotski CC: The role of the unfolded protein response in the heart. J Mol Cell Cardiol 44: 453-459, 2008.

40. Kim I, Xu W and Reed JC: Cell death and endoplasmic reticulum stress: disease relevance and therapeutic opportunities. Nat Rev Drug Discov 7: 1013-1030, 2008.

41. Xu C, Bailly-Maitre B and Reed JC: Endoplasmic reticulum stress: cell life and death decisions. J Clin Invest 115: 2656-2664, 2005.

42. John LM, Lechleiter JD and Camacho P: Differential modulation of SERCA2 isoforms by calreticulin. J Cell Biol 142: 963-973, 1998.

43. Camacho P and Lechleiter JD: Calreticulin inhibits repetitive intracellular $\mathrm{Ca}^{2+}$ waves. Cell 82: 765-771, 1995. 